\title{
Optimization of the storage capacity of slow light photonic crystal waveguides
}

\author{
Panagiotis Kanakis, ${ }^{1, *}$ Thomas Kamalakis, ${ }^{2}$ and Thomas Sphicopoulos ${ }^{1}$ \\ ${ }^{1}$ Department of Informatics and Telecommunications, National and Kapodistrian University/Athens, \\ Panepistimioupolis, Athens GR157 84, Greece \\ ${ }^{2}$ Department of Informatics and Telematics, Harokopio University/Athens, 89 Harokopou Street, Athens GR176 71, Greece \\ *Corresponding author: kanakis@di.uoa.gr
}

Received July 27, 2012; revised September 20, 2012; accepted September 24, 2012; posted September 25, 2012 (Doc. ID 173435); published November 6, 2012

\begin{abstract}
The storage capacity of slow light photonic crystal waveguides is maximized using a systematic procedure based on the optimization of various parameters of the structure. Both optical loss and dispersion-induced broadening are incorporated into the model. The results indicate that this procedure can provide up to a threefold increase in storage capacity. () 2012 Optical Society of America
\end{abstract}

OCIS codes: $130.5296,220.0220$.

Slow light nanophotonic structures such as photonic crystals (PhCs) or coupled resonator optical waveguides (CROWs) [1] are attracting increased attention, combining large slowdown factors, subwavelength light confinement, and enhancement of nonlinear effects [2,3]. However, dispersion and propagation loss limit the performance of these devices, especially in the slow light regime. By intentionally altering the structural characteristics of the $\mathrm{PhC}$ waveguide, several attempts have been made to minimize the group velocity dispersion (GVD) coefficient $\beta_{2}$ [늑] ]. In most works, the proposed structure is identified through exhaustive search, altering only a few design parameters. This is a consequence of the large computational time associated with exhaustive search in a multiparameter space. Also, most works focus on the dispersion characteristics, ignoring the influence of optical loss. Hence, one may obtain structures that achieve large slowdown factors but may exhibit unacceptably high propagation losses, limiting their suitability for delay-line applications. In this work, we show how a $\mathrm{PhC}$ waveguide can be designed systematically, using optimization methods instead of a time-consuming exhaustive search. We carry out our optimization using the actual storage capacity $N_{\max }$ of the delay line at a given data rate $R_{b}$, taking into account both loss and dispersion.

The storage capacity $N_{\max }$ measured in bits can be estimated using the ratio of the achieved delay $L_{W} /\left|v_{g}\right|$ to the bit duration $1 / R_{b}$, i.e.,

$$
N_{\max }=L_{W} R_{b} /\left|v_{g}\right|
$$

where $L_{W}$ is the waveguide length and $v_{g}$ is the group velocity of the defect mode that carries the signal. $N_{\max }$ is therefore a function of $R_{b}$ but also of the wave vector $k$ and the geometry of the waveguide superlattice. Figure 1 illustrates a horizontal cross section of a defect PhC slab waveguide (PCSW) showing some geometry parameters that can be considered in the design. The holes are classified according to their proximity to the waveguide defect (e.g., class "1" denotes the neighbors closest to the waveguide center, and so on). In the $y$ direction, we assume that all the holes of the same class are placed symmetrically with respect to the $x$ axis. In the $x$ direction, the holes located to opposite sides of the $x$ axis can be displaced in either the same (as shown in Fig. 1) or in the opposite direction [4]. Furthermore the radii of the holes in the $i$ th class are all equal to $r_{i}$. Formally we can write $N_{\max }=f\left(R_{b}, a, k, r_{a}, \varepsilon_{a}, \varepsilon_{b}, h, x_{1}, y_{1}, r_{1}, \ldots, x_{N} y_{N} r_{N}\right)$, where $N$ is the number of hole classes considered in the optimization, $r_{a}$ is the radii of the rest of the lattice holes not contained in any of the classes, $a$ is the lattice constant, and $\varepsilon_{a}$ and $\varepsilon_{b}$ are the relative dielectric constants of the high- and low-index material, respectively. The function $f$ is not known in closed form, but as discussed below, it can be computed using a plane wave expansion eigenmode solver [ $\underline{8}]$ to obtain the modal fields and the dispersion relation $k=k(\omega)$ of the waveguide. We can apply standard optimization methods to choose the arguments of $f$ in order to maximize $N_{\max }$ in Eq. (1). Since local optimization depends on the choice of the initial point, a good practice is to carry out the optimization considering several different initial points. In this work we used MATLAB's fmincon function, which is based on an interior-point approach and combines a direct method for solving the constrained minimization problem along with conjugate gradient steps using trust regions [9].

To estimate $N_{\max }$, one must estimate $L_{W}$, which is determined by the maximum tolerable optical loss and the dispersion-induced pulse broadening. In this work, we consider that the loss limit is $l_{\max }=-20 \mathrm{~dB}$, which can be easily compensated by semiconductor optical amplifiers [10]. Given the optical loss coefficient $\Gamma$ of the waveguide in $\mathrm{dB} / \mathrm{cm}$, the maximum propagation distance due to losses is simply $L_{\Gamma}=-l_{\max } / \Gamma$. The loss coefficient, $\Gamma$, can be written as $\Gamma=c_{1} \gamma n_{g}+c_{2} \rho n_{g}^{2}$, where $\gamma$ and $\rho$ are the out-of-plane and backscattering coefficients, $c_{1}$ and $c_{2}$ are constants that describe the technological parameters (independent of design), and $n_{g}$ is the

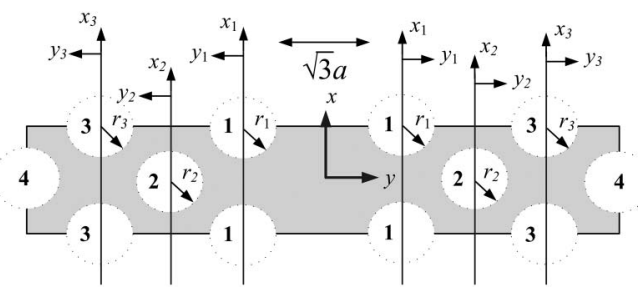

Fig. 1. Illustration of the various geometry design parameters. 
group index [6]. The coefficients $\gamma$ and $\rho$ can be calculated from the modal fields using the expressions provided in [6]. For the waveguide designs considered below, we have verified that backscattering is the dominant optical loss contribution and hence $\Gamma \cong c_{2} \rho n_{g}^{2}$. This simple expression for $\Gamma$ allows us to relate the losses $\Gamma_{\text {ref }}$ of any structure operating at a group index $n_{g}$ to that of a reference waveguide operating at the same $n_{g}$. It can be easily shown that

$$
\Gamma=\Gamma_{\text {ref }}\left(\rho / \rho_{\text {ref }}\right) .
$$

In our calculations, we have used the loss-engineered waveguide of [ 6 ] as a reference, assuming $2 \mathrm{~dB} / \mathrm{cm}$ loss in the fast light regime $\left(n_{g} \cong n_{g 0}=5\right)$. For this design, both the loss and the backscattering coefficient $\rho_{\text {ref }}$ have been well documented and are in good agreement with the loss model of Eq. (2). The backscattering coefficients $\rho$ and $\rho_{\text {ref }}$ can be calculated using the expressions outlined in []ㅡ. Although Eq. (2) ignores the effect of out-of-plane losses, we have later considered the influence of the latter in the capacity of an optimum structure described below. Alternative loss models could also be used in the calculations provided they are not time consuming.

The dispersion-induced broadening factor, i.e., the ratio of the rms width of the pulse at a given position $x$ along the waveguide to the input rms width, can be calculated in closed form [10]. Assuming Gaussian pulses with initial full width at half-maximum $T_{F}=1 /\left(4 R_{b}\right)$, the initial rms width is $\sigma_{0} \cong T_{F} / 2.36$, and we can show that the maximum allowable length due to dispersion $L_{B}$ is

$$
L_{B}=K\left(B_{\max }^{2}-1\right)^{\frac{1}{2}}\left(\beta_{2}^{2} R_{b}^{4}+\frac{1}{4} K^{-1} \beta_{3}^{2} R_{b}^{6}\right)^{-\frac{1}{2}}
$$

where, $\beta_{2}$ and $\beta_{3}$ are the GVD and third-order dispersion coefficients, respectively, $B_{\max }=1.3$ is the maximum allowable broadening factor [10], and $K=0.0224$. The coefficients $\beta_{2}$ and $\beta_{3}$ are obtained through polynomial fitting of the dispersion relation $k=k(\omega)$ of the mode. Note that if $\beta_{2}=0$ and the waveguide is dispersion-limited $\left(L_{W}=L_{B}\right)$, then $N_{\max } \sim L_{W}^{2 / 3}$, as in the case of CROWs [11].

We choose the maximum propagation length $L_{W}$ as the minimum of $L_{B}, L_{\Gamma}$, and $L_{\max }$ where $L_{\max }$ is the waveguide length limit imposed by optical integration considerations. We set $L_{\max }=1 \mathrm{~cm}$, in order to ensure that the waveguide is short enough to be realized in integrated form. Given $L_{W}$, the storage capacity $N_{\max }$ is easily calculated by Eq. (1).

Figure 2 shows the values of $N_{\max }$ as a function of $k$ obtained by optimizing various PCSW designs proposed in the literature [3-6]. The figure also illustrates the wave vector intervals for which the optimized design is dispersion-limited $\left(L_{W}=L_{B}\right)$, loss-limited $\left(L_{W}=L_{\Gamma}\right)$, or limited due to size considerations $\left(L_{W}=L_{\max }\right)$. The optimization process was carried out by altering the same design parameters as proposed in each work, at a bit rate of $R_{b}=$ $40 \mathrm{~Gb} / \mathrm{s}(\mathrm{Gb} / \mathrm{s})$. Figure 2(a) shows the results obtained for the PCSW proposed in []ㅡ, which is realized by perturbing the positions of the neighboring holes by $\Delta y_{1}=$ $0.1171 a$ and $\Delta y_{2}=-0.039 a$. The figure illustrates that the original design corresponds to $N_{\max }=13.4$ bits.
Applying the optimization process we obtain almost double the storage capacity $\left(N_{\max }=25.6\right.$ at $\left.n_{g} \cong 20\right)$ for $\Delta y_{1}=$ $0.1354 a$ and $\Delta y_{2}=0.0436 a$. For these parameters, the waveguide is loss-limited $\left(L_{W}=L_{\Gamma} \cong 9.6 \mathrm{~mm}\right)$. In Fig. 2(b), we show the capacity obtained by optimizing the structure of [5]. The original design was obtained through exhaustive search by altering $x_{1}$ and $y_{2}$. The $x$ positions of the holes on opposite sides of the $x$ axis are changed in opposite directions. The optimum dispersion delay bandwidth product (DBP) is obtained for $\Delta x_{1}=0.105 a$ and $\Delta y_{2}=-0.084 a$ without considering the effect of optical losses. Figure $2(\mathrm{~b})$ suggests that this design corresponds to $N_{\max }=\overline{8.3}$ bits. Applying the optimization procedure we obtain a size-limited design $\left(L_{W}=L_{\max }=1 \mathrm{~cm}\right)$ with $N_{\max } \cong 12$ at $n_{g}=9$ for $\Delta x_{1}=$ $0.0302 a$ and $\Delta y_{2}=-0.0287 a$. Figure $2(\mathrm{c})$ discusses the improvement brought about in the air-cladding waveguide design of [4], which maximizes the DBP, perturbing the hole positions by $\Delta y_{1}=0.1208 a$ and $\Delta y_{2}=0.029 a$, achieving a capacity of $N_{\max }=10.4$ bits. The optimization process yields a loss-limited design with $N_{\max }=$ 17.7 bits at $n_{g}=24$, for $\Delta y_{1}=0.1257 a$ and $\Delta y_{2}=0.04 a$. Finally, Fig. 2(d) shows the structure proposed by []], setting $\Delta x_{3}=\overline{0.32} a$ for an air-cladding chalcogenide PCSW corresponding to $N_{\max }=13.2$ bits. The holes are moved in the $x$ axis as shown in Fig. 1. The optimization process yields a dispersion-limited design with $N_{\max }=16.7$ bits at $n_{g}=22$, obtained for $\Delta x_{3}=0.3 a$. Note that this result is obtained assuming the same loss level as in silicon, although chalcogenide waveguides are inherently more lossy [7]. If one assumes a more realistic loss level (more than a tenfold fast light loss compared to silicon), then changing $x_{3}$ does not yield any increase in $N_{\max }$ since it does not affect the loss and the waveguide remains loss limited, even at moderate values of $n_{g}$.

The results presented thus far indicate that the optimization process can be used in order to maximize $N_{\max }$, taking into account the effects of optical losses and dispersion-induced broadening in a rigorous manner. It is also interesting to note that as shown in Fig. $\underline{2}$, the optimum $N_{\max }$ corresponds to a point in the dispersion relation near the transition from one length-limiting factor to another. For example, in the designs of
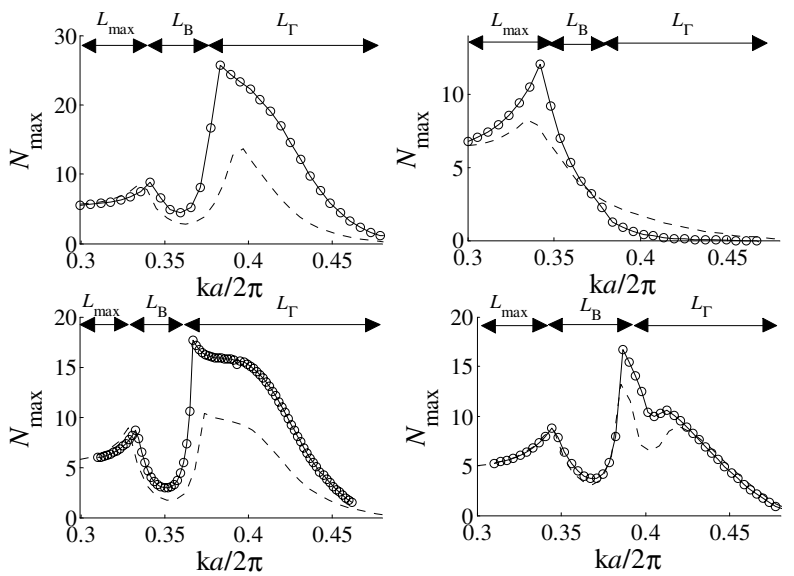

Fig. 2. Capacity of the original structures (curve only) proposed by (a) O'Faolain et al. [6] ], (b) Liang et al. [5], (c) Li et al. in [4] , and (d) Suzuki and Baba [ []] and the structures obtained by optimization (curve with open circle). 
Figs. 2(c) and 2(d) we obtain $L_{B} \cong L_{\Gamma}$, indicating that loss and dispersion are equally important as in the case of CROWs [12].

We now discuss how the optimization procedure can be applied in order to design a PCSW delay line from scratch, considering the effect of multiple design parameters. We choose the standard $\mathrm{W} 1$ waveguide as a starting point with $r_{a}=0.27 a$, and perform a step-by-step optimization gradually increasing the number of parameters considered. The capacity of the original waveguide is shown in Fig. 3. The figure also shows the capacity obtained by first optimizing the structure with respect to $y_{1}$ and $k$, which yields $N_{\max } \cong 23$ for $\Delta y_{1}=0.1345 a$. The second optimization step uses as a starting point the parameters obtained in the first step but also considers $y_{2}$ in addition to $y_{1}$ and $k$. The capacity now reaches $N_{\max }=25.6$ for $\Delta y_{1}=$ $0.1354 a$ and $\Delta y_{2}=0.0436 a$. In the third optimization step, we are optimizing with respect to $\Delta y_{3}$ as well, which does not yield significant improvement, bringing the maximum capacity to $N_{\max }=26.7$. Since the change was small, we have not included the curve of the third step in Fig. 3. In the fourth step, we have also considered the radius perturbations $\Delta r_{1}$, and the optimization yields a loss-limited design with $N_{\max }=31.3$ at $n_{g} \cong 24$ for $\Delta y_{1}=0.1297 a$, $\Delta y_{2}=-0.0248 a, \quad \Delta y_{3}=0.0399 a$, and $\Delta r_{1}=0.25 a$. Figure 3 also includes the dispersion relation of this waveguide design and the group index. Interestingly enough, a flat group index is obtained for an $11 \mathrm{~nm}$ wavelength range where the group index variation is less than $10 \%$ around $n_{g} \cong 24$. This is consistent with the relatively smooth variation of $N_{\max }$ with respect to $k$ around $k a /(2 \pi)=0.39$, suggesting the suitability of this design for wavelength division multiplexing applications, although no specific measures have been taken to ensure this during the optimization process. Monomode operation is guaranteed for a wide frequency range around the optimum $k$ point in every step of the optimization process. Rounding the parameters of the optimum design up to a second decimal digit (e.g., $\Delta y_{1}=0.13 a$ and so forth), we obtain a negligible change in $N_{\max }(<1 \%)$, indicating that the design is insensitive to small variations due to limited fabrication resolution. This also holds for the rest of the designs. For example, in the case of the design in Fig. 2(a), the corresponding change in $N_{\max }$ is less than $3 \%$.

Thus far, our analysis neglected the effect of out-of-plane scattering. We also examined an alternative loss model $\Gamma^{\prime}$, where out-of-plane scattering is no longer negligible and is assumed to have equal contribution to the losses in the fast lightregime $\left(n_{g}=n_{g 0}\right)$. Under these assumptions, it is easy to see that $\Gamma^{\prime}=1 / 2 \Gamma+1 / 2 \Gamma_{0}\left(\gamma n_{g}\right) /\left(\gamma_{0} n_{g 0}\right)$, where the second term corresponds to the out-of-plane losses, $\gamma=\gamma\left(n_{g}\right)$ is the out-of-plane scattering coefficient [] $], \gamma_{0}=\gamma\left(n_{g 0}\right)$, and $\Gamma_{0}=\Gamma\left(n_{g 0}\right)=\Gamma^{\prime}\left(n_{g 0}\right)$ is the lossin the fastlight regime assumed equal for both models. Figure 3 includes the capacity obtained for $\Gamma^{\prime}$, which is marginally higher since the out-of-plane loss part is proportional to $n_{g}$ rather than $n_{g}^{2}$, and hence the total losses are lower in the slow light regime.

Considering the rest of the design parameters $x_{1}, x_{2}$, $x_{3}, r_{2}$, and $r_{3}$, only a marginal improvement was obtained, $N_{\max } \cong 33$. It should be mentioned that the full optimization process including all nine steps involved about 10 iterations per step and each iteration required $10-15$ function evaluations, resulting in no more than 1350

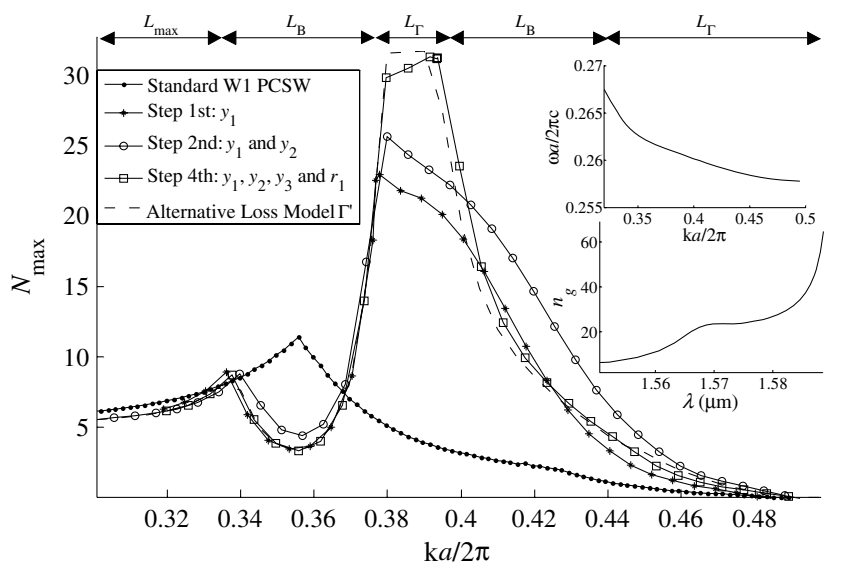

Fig. 3. Storage capacity obtained in various optimization steps. The subfigures show the mode dispersion relation and the group index of the optimum design.

evaluations in total. Finally, we examined the case of $R_{b}=100 \mathrm{~Gb} / \mathrm{s}$, where the maximum capacity obtained was $N_{\max } \cong 65$ at $n_{g} \cong 21$ for $\Delta y_{1}=0.14 a, \Delta y_{2}=0.025 a$, $\Delta y_{3}=0.018 a, \Delta r_{1}=0.26 a$, and $L_{W}=L_{\Gamma}=9.5 \mathrm{~mm}$. In conclusion, we have presented an optimization methodology for slow light photonic crystal waveguides that takes into account both dispersion and loss-induced effects. We have shown that the optimization can increase the storage capacity of known designs and may also be used to design a slow light waveguide from scratch.

This research has been cofinanced by the European Union [European Social Fund (ESF)] and Greek National Funds through the Operational Program "Education and Lifelong Learning" of the National Strategic Reference Framework (NSRF)-Research Funding Program: Heracleitus II. Investing in knowledge society through the European Social Fund.

\section{References}

1. A. Melloni, A. Canciamilla, C. Ferrari, F. Morichetti, L. O'Faolain, T. F. Krauss, R. De La Rue, A. Samarelli, and M. Sorel, IEEE Photonics J. 2, 181 (2010).

2. A. E. Willner and S. R. Nuccio, Proc. SPIE 7949, 794908 (2011).

3. C. Monat, B. Corcoran, M. Ebnali-Heidari, C. Grillet, B. J. Eggleton, T. P. White, L. O'Faolain, and T. F. Krauss, Opt. Express 17, 2944 (2009).

4. J. Li, T. P. White, L. O'Faolain, A. G. Iglesias, and T. F. Krauss, Opt. Express 16, 6227 (2008).

5. J. Liang, L. Ren, M. Yun, X. Han, and X. Wang, J. Appl. Phys. 110, 063103 (2011).

6. L. O'Faolain, S. A. Schulz, D. M. Beggs, T. P. White, M. Spasenovic, L. Kuipers, F. Morichetti, A. Melloni, S. Mazoyer, J. P. Hugonin, P. Lalanne, and T. F. Krauss, Opt. Express 18, 27627 (2010).

7. K. Suzuki and T. Baba, Opt. Express 18, 26675 (2010).

8. S. G. Johnson and J. D. Joannopoulos, Opt. Express 8, 173 (2001).

9. R. H. Byrd, M. E. Hribar, and J. Nocedal, SIAM J. Optimizat. 9, 877 (1999).

10. R. Ramaswami and K. N. Sivarajan, Optical Networks: A Practical Perspective (Morgan Kaufman, 1998).

11. J. B. Khurgin, J. Opt. Soc. Am. B 22, 1062 (2005).

12. J. B. Khurgin, Opt. Lett. 32, 133 (2007). 